\title{
Hereditary Nephritis and Thin Glomerular Basement Membrane Lesion
}

\author{
Mark A. Lusco Agnes B. Fogo \\ Department of Pathology, Microbiology and Immunology, Vanderbilt University Medical Center, Nashville, TN, USA
}

\section{Key Points}

- Clinical history.

- Thin glomerular basement membrane by EM.

- Collagen type IV subtype staining pattern by IF.

\section{Keywords}

Hereditary nephritis - Thin glomerular basement membrane $\cdot$ Alport syndrome $\cdot$ Type IV collagen

\section{Abstract}

Hereditary nephritis $(\mathrm{HN})$ and thin glomerular basement membrane (GBM) lesion share a common clinical presentation of persistent hematuria, thin GBM by kidney biopsy electron microscopic examination, and a mutation in type IV collagen. However, the clinical course and treatment for these entities are different with varying patterns of heredity. Ultrastructural examination of a renal biopsy specimen is essential for the morphologic diagnosis of HN and thin GBM lesion, whereas light microscopy may only give limited diagnostic clues. Additional workup including immunostaining for subtypes of type IV collagen may provide further information on underlying genetic mutations. The diagnosis of $\mathrm{HN}$ may lead to treatment with renin-angiotensin system blockade in patients at risk of early-onset renal failure to delay progression to end-stage renal disease. Additionally, patients with isolated microscopic hematuria and thin GBM lesion are at in-

karger@karger.com www.karger.com/gdz

Karger ${ }^{\prime \prime} \div$
C 2021 The Author(s)

Published by S. Karger AG, Basel

This is an Open Access article licensed under the Creative Common Attribution-NonCommercial-4.0 International License (CC BY-NC) (http://www.karger.com/Services/OpenAccessLicense), applicable to the online version of the article only. Usage and distribution for commercial purposes requires written permission. creased risk for chronic kidney disease when associated with other comorbidities; those patients should receive regular clinical assessment to prevent renal function decline.

(c) 2021 The Author(s)

Published by S. Karger AG, Basel

\section{Introduction}

Hereditary nephritis (HN), also known as Alport syndrome, is defined as a spectrum of disease caused by mutations in genes encoding for type IV collagen and resulting in hematuria, progressive renal dysfunction with eventual proteinuria, deafness, and vision changes. Thin glomerular basement membrane (GBM) lesion (also known as thin basement membrane disease, thin basement membrane nephropathy, and benign familial hematuria) shares a clinical presentation of hematuria. Many patients also are heterozygous for mutations in the collagen IV gene (autosomal recessive). However, these heterozygous patients with thin GBM lesion do not show deafness or ocular changes. In the past, thin GBM lesion 
was not thought to lead to chronic kidney disease; a view that has changed in modern days since disease progression and development of hypertension are now recognized as long-term complications [1].

\section{Pathophysiology}

$\mathrm{HN}$ occurs as a result of a mutation in type IV collagen involving subtypes alpha 3 , alpha 4 , or alpha 5 . One or more mutations result in a defective assembly of the alpha 3,4 , and 5 collagen IV heterotrimer that is essential for the integrity of GBMs, eye lenses, and ear cochlea structures, and the corresponding functional properties at these sites. The X-linked form of $\mathrm{HN}$ is the result of a mutation in the COL4A5 gene, leading to abnormal or even absent collagen IV alpha 5 chain. Autosomal forms of $\mathrm{HN}$ are the result of a mutation in COL4A3 or COL4A4 resulting in abnormal COL4 assembly and distortion of GBM architecture [2-4].

Thin GBM lesion is most commonly the result of an autosomal recessive COL4A3 or COL4A4 mutations. Of note, thin GBM can also be an initial early finding in some patients developing Alport syndrome later on and in heterozygote females with X-linked COL4A5 mutations [2-4].

\section{Clinical Manifestations}

Previous estimates indicated X-linked Alport accounts for about $80 \%$ of Alport syndrome, autosomal recessive Alport for about 15\%, and autosomal dominant Alport for the remainder of cases. Next generation sequencing shows that autosomal dominant Alport is more frequent than previously though; in 1 study this pattern was present in up to $\sim 30 \%$ of the families with suspected Alport where a COL4 mutation was detected $[5,6]$. X-linked Alport presents in infancy with hematuria in all the afflicted males, and also in nearly all heterozygote females. Proteinuria develops in the second decade due to the sclerosis occurring in glomeruli with "Alport-changes" and may present as nephrotic syndrome in some patients (30$40 \%)$ during further disease progression. Some heterozygote females may develop proteinuria in later stages. Therefore, it is not surprising that COL4A mutations are seen in some patients primarily presenting at the time of biopsy with significant proteinuria and segmental glomerular tuft sclerosis [7]. It is important to note that such patients do not have "conventional focal segmental glomerulosclerosis," but rather a sclerosing hereditary glo-
merulopathy/Alport disease characterized ultrastructurally by typical GBM abnormalities. This patient cohort underscores the need and significance of electron microscopy in the workup of renal biopsies. Alport syndrome patients have sensorineural deafness (eventually in $90 \%$ of males and $40 \%$ by age 40 ) and decreased visual acuity ( $20 \%$ of males, rare in females but with increased occurrence by age 60) related to bilateral anterior lenticonus, anterior polar cataract, retinal flecks, and retinal and iris neovascularization. There is no specific treatment for $\mathrm{HN}$ and patients progress to end-stage renal disease (all males by age 60$)[8,9]$. The patients receiving a transplant kidney generally do well, as there is no recurrence of the disease in the allograft. However, in the kidney transplant recipients, there is a rare occurrence (2-5\%) of anti-GBM antibody disease caused by a de novo antibody response to new (and normal) GBM components in the allograft kidney. Once Alport patients have experienced such antiGBM antibody disease in the transplant, this complication occurs in the subsequent transplants [10].

Thin GBM lesion presents with persistent microscopic hematuria with intermittent macroscopic hematuria, and generally has a benign clinical course when caused by the heterozygous state of an autosomal COL4 mutation. There is no specific treatment. The patients homozygous or compound heterozygous for autosomal recessive, $\mathrm{Al}$ port may show some degree of the renal dysfunction in early adulthood, accompanied in some patients by moderate proteinuria [11]. The patients with autosomal dominant Alport syndrome may show renal dysfunction in adulthood [12]. Thin GBM may also be present in some patients with loin pain hematuria syndrome, without any known mutations described in this condition so far. These patients also have increased risk factors for stones [13].

In patients with $\mathrm{HN}$ who are at risk for early-onset renal failure, treatment with renin-angiotensin system blockade can delay the onset of end-stage renal disease. [14-16]. Also, the patients with isolated microscopic hematuria and thin GBM lesions are at risk for chronic kidney disease especially in the setting of other comorbidities, such as hypertension. Those patients can benefit from close monitoring to prevent progressive renal failure $[1,16]$.

\section{Light Microscopy}

$\mathrm{HN}$ in the very early stage is unremarkable by LM, although there may be intratubular red blood cell casts in few patients or the interstitial foam cells. In later stages of 


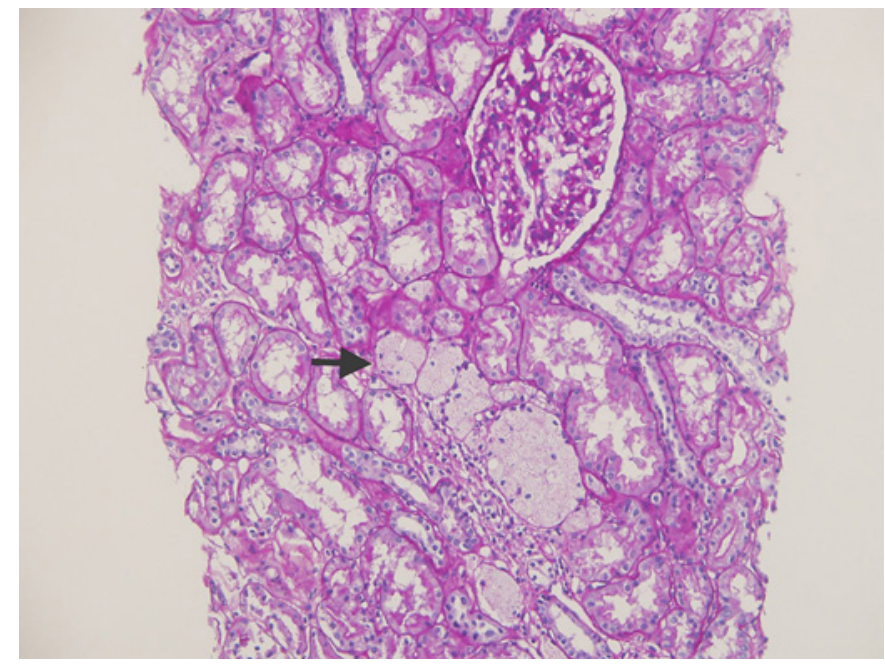

Fig. 1. X-linked Alport syndrome in a male patient with an unmarkable glomerulus and interstitial foam cells (arrow) (Periodic acid-Schiff stain $\times 200$ ).

the disease, the interstitial foam cells are often conspicuous. During further progression "chronic changes" become more prominent with secondary global and segmental glomerulosclerosis, increasing interstitial fibrosis, and tubular atrophy (shown in Fig. 1, 2). Note: HN foam cells are seen in patients without or only subnephrotic range proteinuria, likely reflecting the long duration of this lesser magnitude of proteinuria. In contrast, the foam cells are often seen with other renal diseases associated with nephrotic range proteinuria. Thin GBM lesion shows unremarkable LM except for possible intratubular red blood cell casts (shown in Fig. 3).

\section{Immunofluorescence Microscopy}

Immunofluorescence (or immunohistochemistry) for collagen staining of the kidney biopsies can be used to help distinguish potential genetic abnormalities underlying an abnormal GBM ultrastructural appearance. The pattern of staining for the COL4 alpha chain subtypes depends upon the underlying gene mutation. Of note, collagen staining may be normal in some instances with rare mutations, such as some missense mutations. In HN, per se immune complexes are not found, although occurrences of superimposed glomerulonephritis in addition to Alport disease have been reported [17].

Alpha 1 or alpha 2 type IV collagen is used as a tissue control, staining all the basement membranes. The nor-

Hereditary Nephritis and Thin

Glomerular Basement Membrane

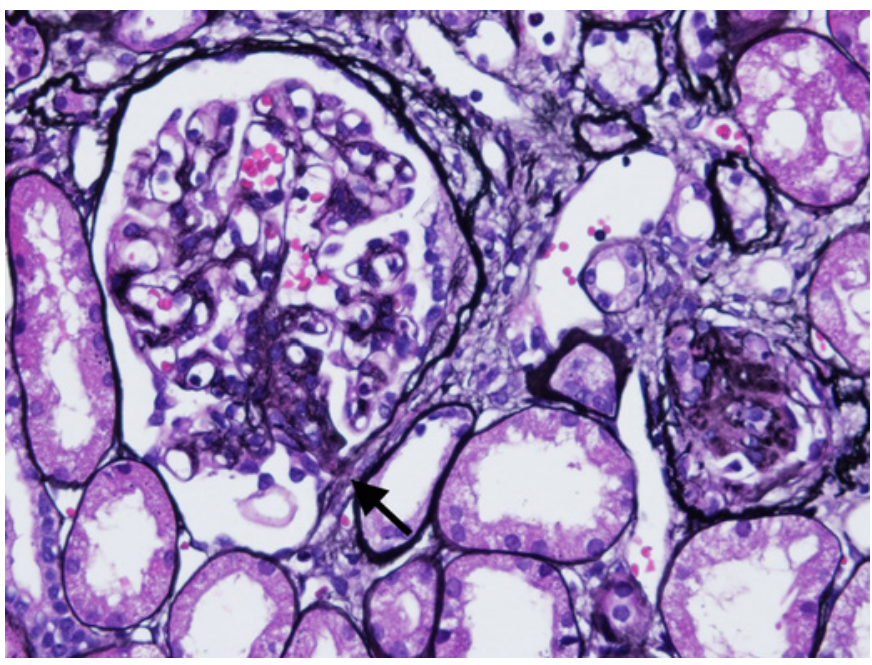

Fig. 2. Segmental adhesion and glomerulosclerosis (arrow) in a male patient with X-linked Alport syndrome (Jones silver stain $\times 400)$.

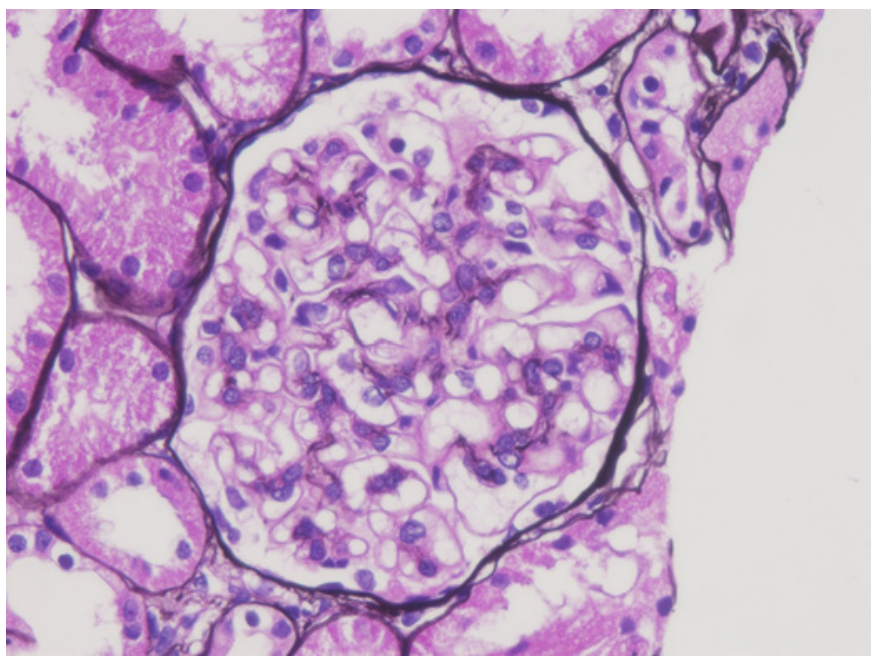

Fig. 3. Unremarkable glomerulus by LM with thin GBM lesion by EM (Jones silver stain $\times 400$ ). GBM, glomerular basement membrane

mal immunofluorescence staining pattern for subtypes of type IV collagen is continuous along glomerular and distal tubular basement membranes (containing collagen type IV heterotrimer alpha 3,4, and 5), and continuous along Bowman's capsule (containing collagen type IV heterotrimer alpha 5, 5, and 6; shown in Fig. 4). X-linked Alport males show absence of alpha 5 type collagen IV staining with no glomerular basement membrane and

Glomerular Dis 2021;1:135-144 


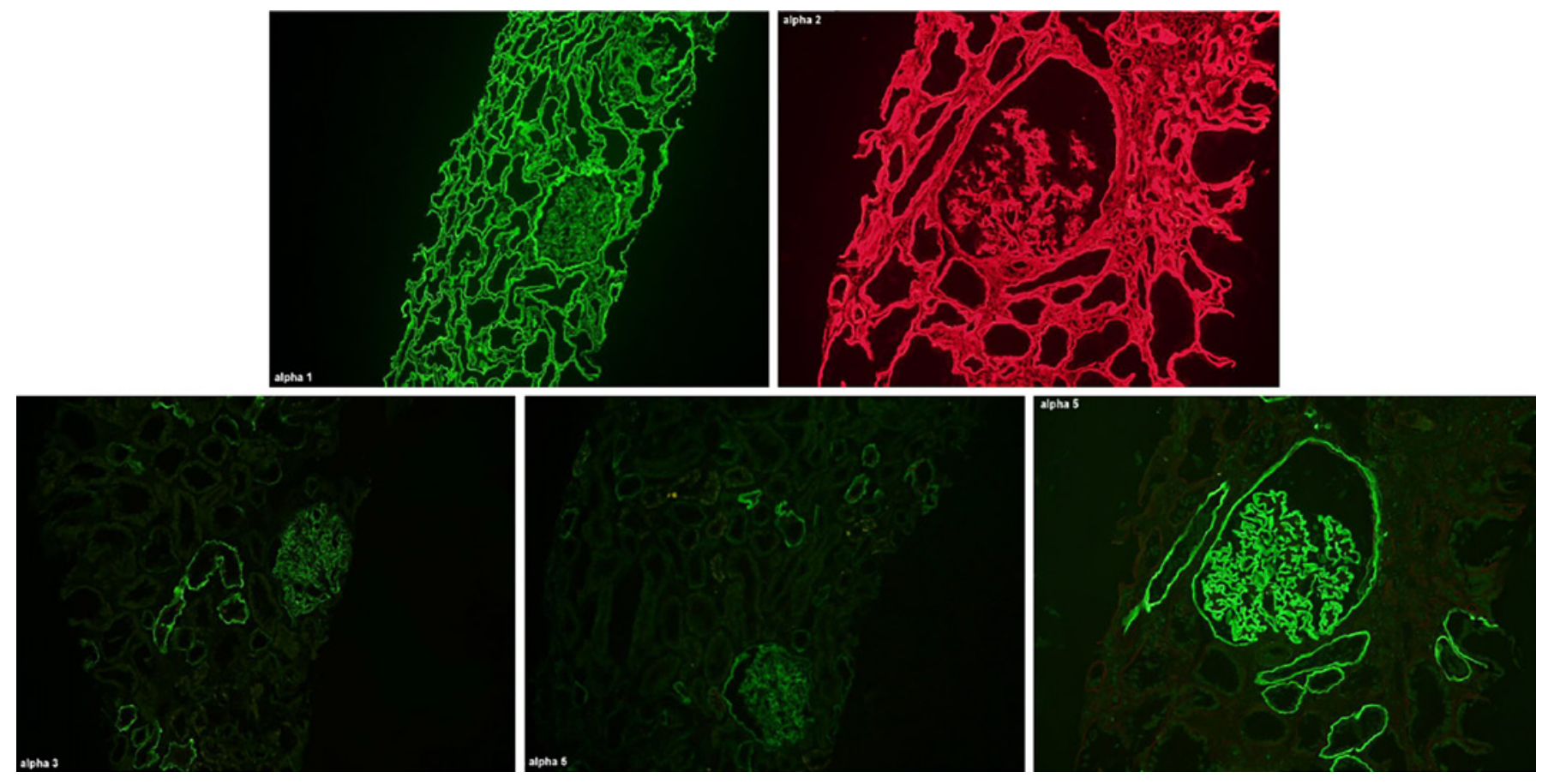

Fig. 4. Normal pattern of staining for subtypes of type IV collagen. Alpha 1 and alpha 2 type IV collagens are present in all basement membranes. Alpha 3 type IV collagen is present in GBMs and focal distal tubule basement membranes. Alpha 5 type IV collagen is present in GBMs, Bowman's capsule, and focal distal tubule basement membranes (immunofluorescence, FITC-conjugated anti- alpha 1 type IV collagen $\times 200$, Texas Red-conjugated anti-alpha 2 type IV collagen, $\times 400$, FITC-conjugated anti-alpha 3 type IV collagen $\times 200$, FITC-conjugated anti-alpha 5 type IV collagen $\times 200$, FITC-conjugated anti-alpha 5 type IV collagen $\times 400$ ). GBMs, glomerular basement membranes.
Bowman's capsule or distal tubule staining (shown in Fig. 5). Heterozygote females with X-linked Alport show a mosaic discontinuous pattern of GBM staining for alpha 5 type collagen IV due to the Lyon effect, with normal or mutated alleles activated in the individual cells (shown in Fig. 6) $[2,8,18]$.

Autosomal recessive Alport homozygous or compound heterozygous male or female patients show the absence of glomerular basement membrane staining for alpha 3 and alpha 5 type IV collagen with maintained continuous Bowman's capsule and tubular basement membrane staining for alpha 5 type IV collagen staining, due to the presence of the alpha 5, 5, and 6 heterotrimer, not requiring intact alpha 3 or 4 for assembly at these latter sites (shown in Fig. 7). Autosomal dominant Alport shows a normal pattern of staining for subtypes of type IV collagen in the rare cases described and therefore, next generation sequencing may be necessary to make this diagnosis.

The epidermal basement membrane contains the collagen type IV heterotrimer alpha 5, 5, and 6. Therefore, staining for alpha 5 collagen type IV is useful in the diagnosis of X-linked Alport syndrome, but not useful for autosomal forms because alpha 3 or 4 type IV collagens are not expressed in the skin. Male X-linked Alport syndrome patients show absent staining in the skin for alpha 5, while females heterozygous for X-linked Alport show discontinuous alpha 5 staining [2].

Previously, a commercial antibody kit from Wieslab was most commonly used to detect alpha 3 and alpha 5 with alpha 1 staining as the tissue control. However, this antibody set is no longer commercially available and currently often an antibody cocktail directed against COL4A2 serves as the control, with additional detection of alpha 5 type IV collagen [19]. In addition, diagnostic genetic testing is rapidly evolving and costs are decreasing to allow for specific detection of mutations associated with HN. Heterozygous patients with autosomal recessive Alport syndrome show thin GBM and normal pattern staining for subtypes of type IV collagen and no immune complex deposits by routine studies. 


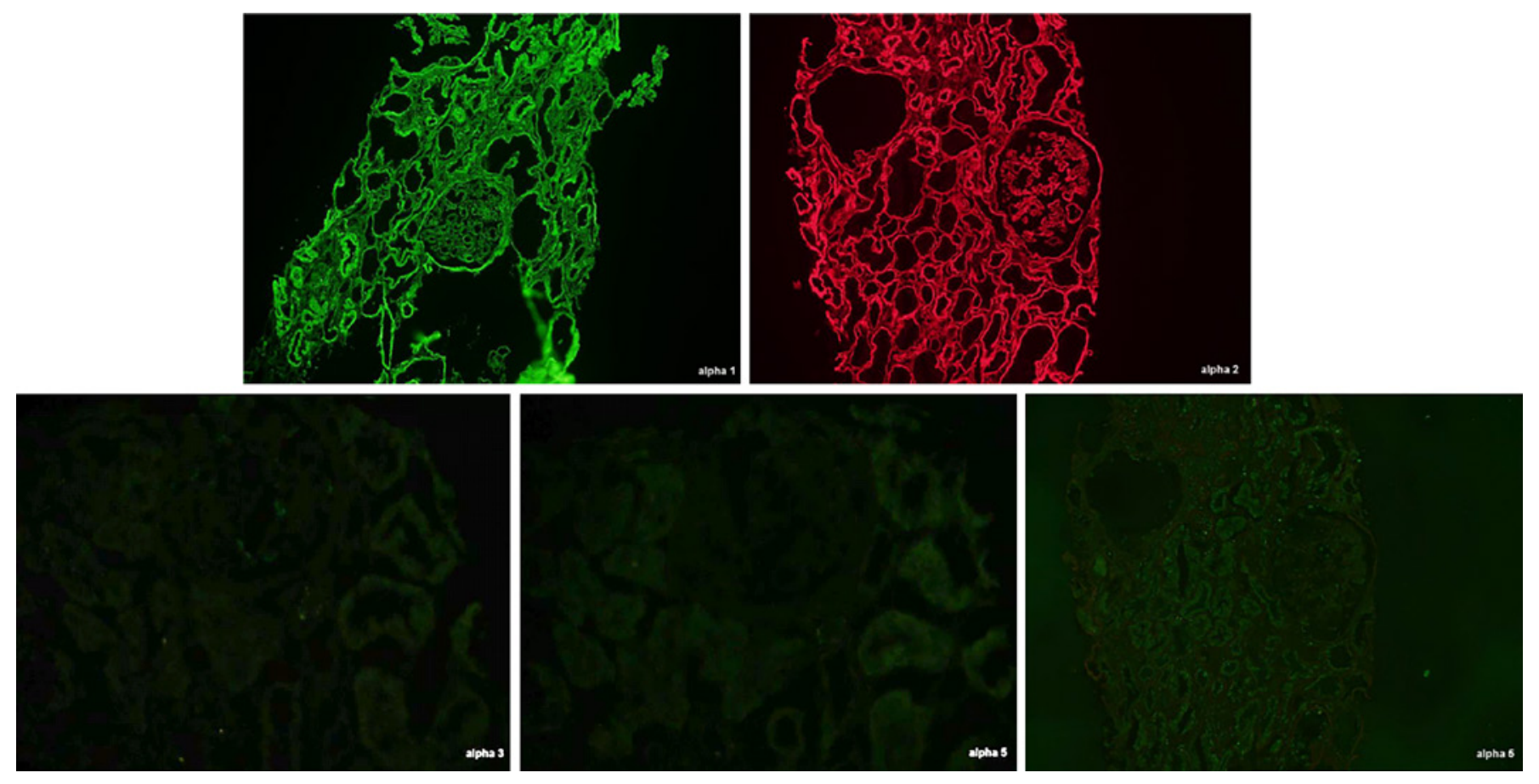

Fig. 5. X-linked Alport syndrome pattern of staining for subtypes of type IV collagen in a male patient. Alpha 1 and alpha 2 type IV collagens are present in all basement membranes showing the tissue is adequately preserved for these studies. Alpha 3 and alpha 5 type IV collagens are absent (immunofluorescence FITC-conju-

\section{Electron Microscopy}

GBM thickness increases with maturational growth, and normal must therefore be compared to normal for age. A diagnosis of thin GBM is generally accepted if $>50 \%$ of the lamina densa is thinner than what can be expected for the patient's age. Of note: the thickness of the GBM is also influenced by the tissue processing, including the choice of fixative and embedding compound for electron microscopy [17]. GBM thickness can only be assessed by ultrastructural analysis, ideally on the glutaraldehyde-fixed tissue. The tissue salvaged for the electron microscopy and reprocessed from formalin-fixed paraffin-embedded tissue may be adequate for the detection of electron-dense deposits and the degree of foot process effacement. However, reprocessing of formalin-fixed and paraffin-embedded tissue results in artifactual GBM thinning and precludes accurate diagnosis of a thin GBM (Fig. 8) [20]. In our laboratory with standard EM processing, normal GBM thickness for a 1 year old is about 110 $\mathrm{nm}$, and for a 7 year old is about $220 \mathrm{~nm}$. For adults, normal ranges are $326 \pm 45 \mathrm{~nm}$ in adult women and $370 \pm 42$

Hereditary Nephritis and Thin

Glomerular Basement Membrane gated anti-alpha 1 type IV collagen $\times 200$, Texas Red-conjugated anti-alpha 2 type IV collagen, $\times 200$, FITC-conjugated anti-alpha 3 type IV collagen $\times 200$, FITC-conjugated anti-alpha 5 type IV collagen $\times 200$, FITC-conjugated anti-alpha 5 type IV collagen $\times 200$ ).

$\mathrm{nm}$ in adult men, with slightly varying ranges in different series (Fig. 9). Thin GBM is considered when the GBM is $<250 \mathrm{~nm}$ in adults and $<180 \mathrm{~nm}$ in children aged 2-11 years in greater than half of the extent of the GBM.

Several methods for measuring GBM thickness have been used, including adding latex beads of known diameter onto the tissue section, using a superimposed grid of known dimensions onto an electron micrograph, using the orthogonal intercept to calculate the harmonic mean of the thickness, and measurement with a metric ruler placed over an electron micrograph $[17,21]$. With the advent of the digital camera, direct measurement of GBM thickness has become the preferred method. Measurements should be made from the endothelial cell membrane closest to the GBM to the podocyte cell membrane in peripheral loops in non-tangential cross sections. The general consensus approach of measuring the GBM thickness requires multiple representative measurements from many peripheral capillary loops free from involvement by other superimposed glomerular disease and calculating the arithmetic or harmonic mean $[17,21]$. Even at the earliest stages of X-linked Alport syndrome, in patients 

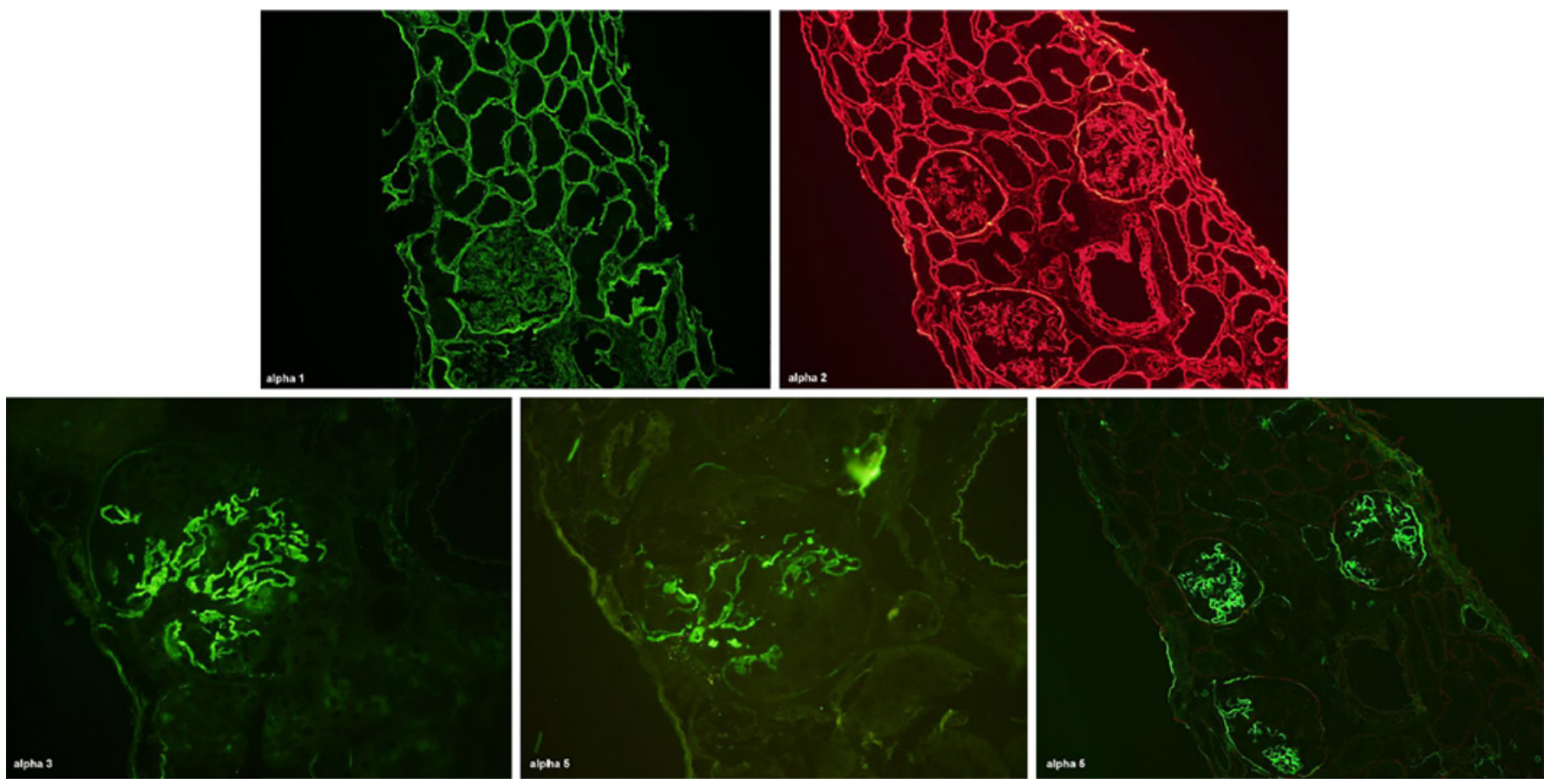

Fig. 6. X-linked Alport heterozygous affected female with mosaic pattern of GBM staining for subtypes of type IV collagen. Alpha 1 and alpha 2 type IV collagens are present in all basement membranes showing the tissue is adequately preserved for these studies. Alpha 3 and alpha 5 type IV collagens show mosaic discontinuous staining along GBMs and some Bowman's capsules (immunofluo- rescence FITC-conjugated anti-alpha 1 type IV collagen $\times 200$, Texas Red-conjugated anti-alpha 2 type IV collagen, $\times 200$, FITCconjugated anti-alpha 3 type IV collagen $\times 400$, FITC-conjugated anti-alpha 5 type IV collagen $\times 400$, FITC-conjugated anti-alpha 5 type IV collagen $\times 200$ ). GBM, glomerular basement membrane.

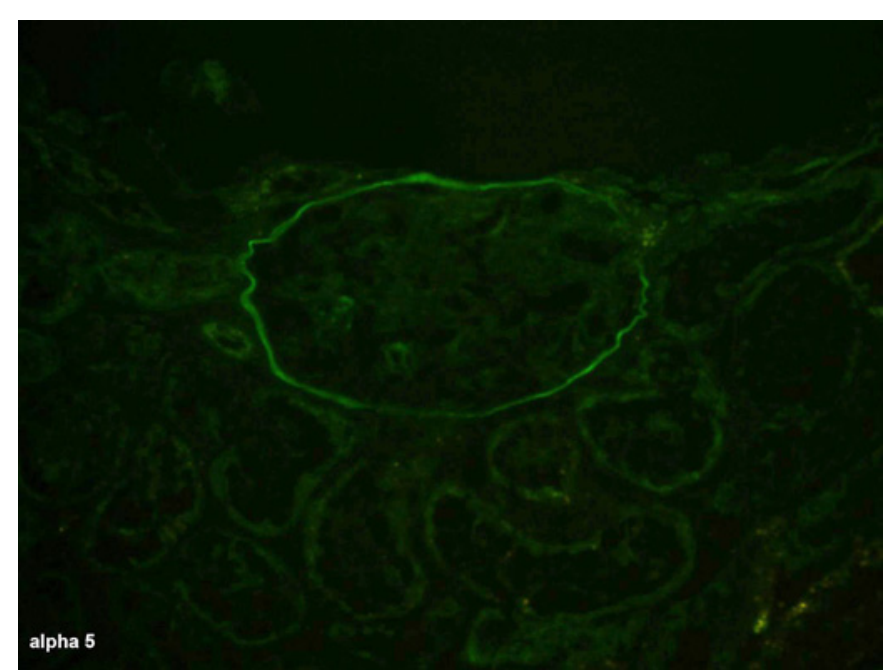

Fig. 7. Autosomal recessive Alport pattern of staining for type IV collagen in a male with absent alpha 5 in GBM but present in Bowman's capsule (immunofluorescence FITC-conjugated anti-alpha 5 type IV collagen $\times 400$ ). GBM, glomerular basement membrane.

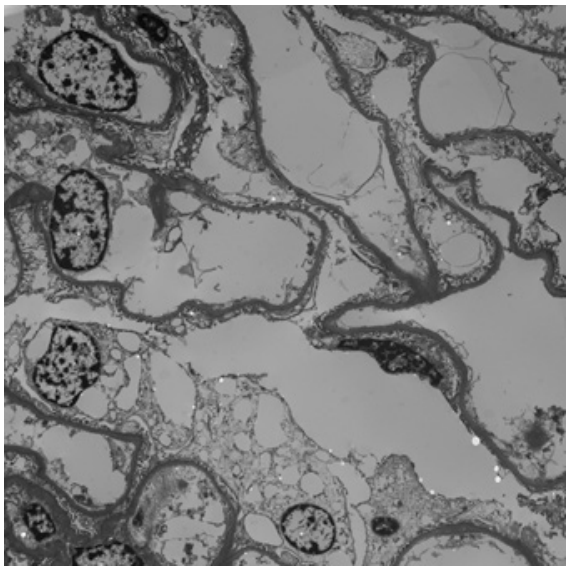

Fig. 8. Deparaffinization of formalin-fixed paraffin-embedded tissue for tissue salvage for EM causes artifactually thin GBM (transmission electron microscopy $\times 2,800$ ). GBM, glomerular basement membrane. 

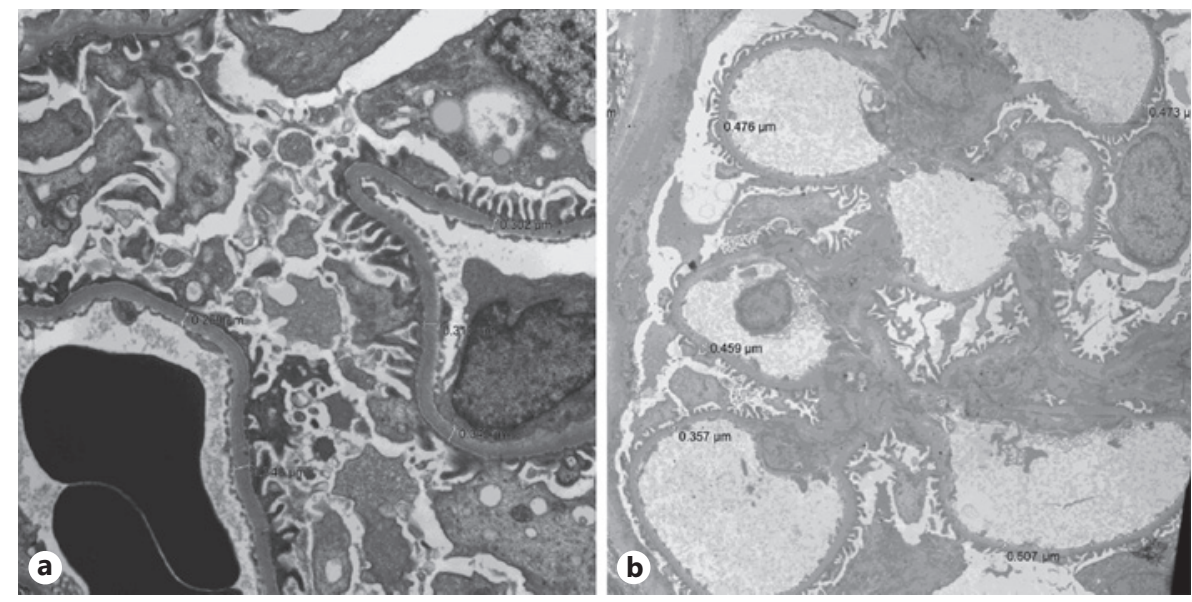

Fig. 9. Normal GBM thickness for adult female $(326 \pm 45 \mathrm{~nm})$ (a, transmission electron microscopy, $\times 4,400)$ and male patient $(370 \pm 42 \mathrm{~nm})(\mathbf{b}$, transmission electron microscopy, $\times 2,200)$. GBM, glomerular basement membrane.

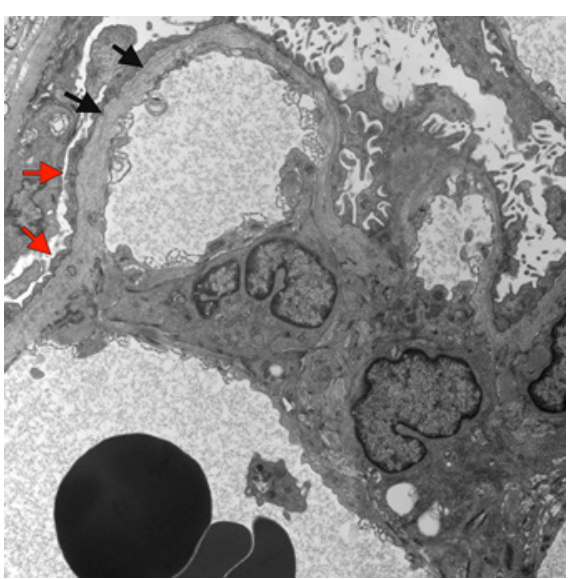

Fig. 11. X-linked Alport syndrome in a male patient with segmental lamellation of the GBM (thin areas $\sim 90 \mathrm{~nm}$, thickened lamellated areas $\sim 500 \mathrm{~nm}$ ) (black arrows) with moderate foot process effacement (red arrows) (transmission electron microscopy $\times 4,400)$. GBM, glomerular basement membrane.

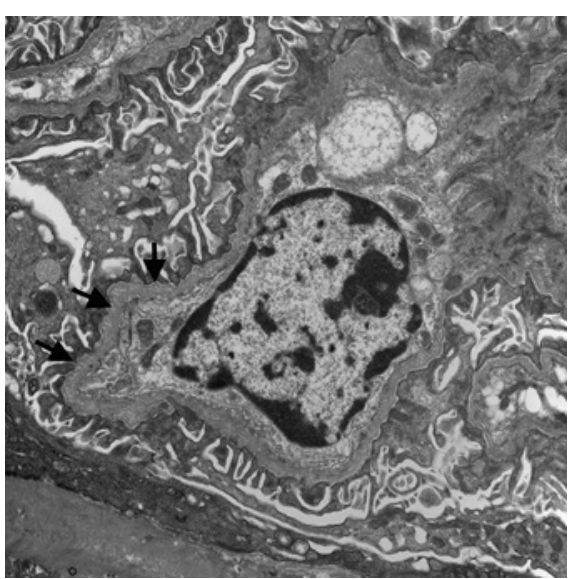

Fig. 12. X-linked Alport syndrome in a heterozygous female patient with thin GBM (segmental $\sim 130 \mathrm{~nm}$ ) with segmental basket-weave appearance ( $400 \mathrm{~nm}$ ) (arrows) with limited foot process effacement (transmission electron microscopy $\times 7,100)$. GBM, glomerular basement membrane.

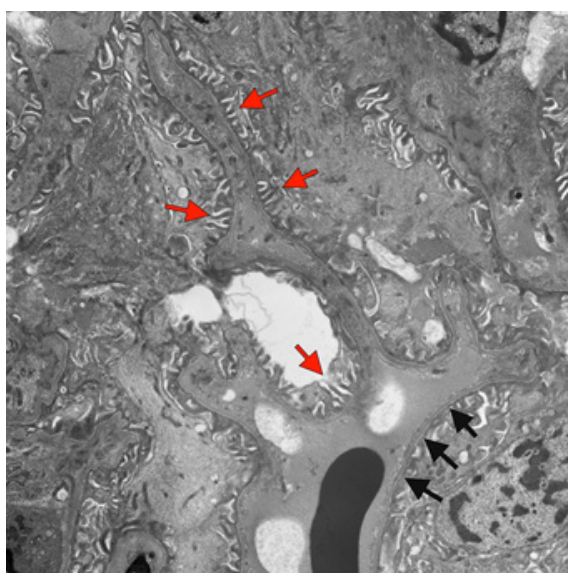

Fig. 10. X-linked Alport syndrome in a female patient with diffusely thin GBM $(\sim 80$ $\mathrm{nm}$ ) (black arrows with largely intact foot processes (red arrows) (transmission electron microscopy $\times 4,400)$. GBM, glomerular basement membrane.

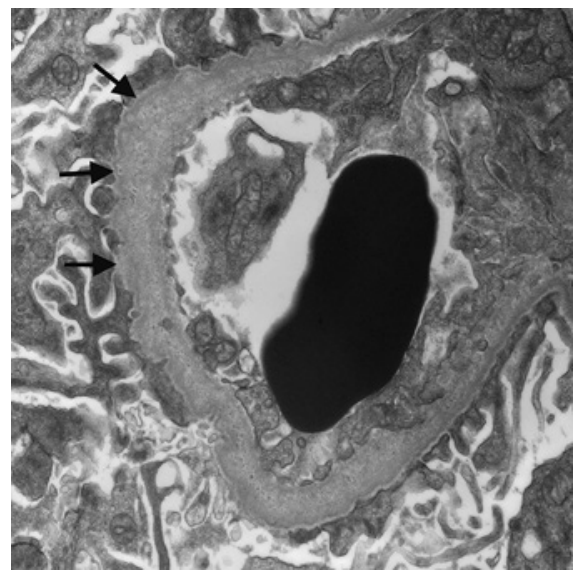

Fig. 13. Autosomal recessive Alport syndrome in a male patient with segmental mottling ( $600 \mathrm{~nm}$ ) (arrows) of the GBM (transmission electron microscopy $\times 7,100)$. GBM, glomerular basement membrane. with autosomal forms of $\mathrm{HN}$, and in those heterozygous for AR gene mutations, thinning of the GBM/lamina densa is a constant finding (shown in Fig. 10).

As $\mathrm{HN}$ progresses in male $\mathrm{X}$-linked Alport patients and afflicted autosomal patients, the GBM becomes irregular with thin and thick segments and an irregular, multi-lam- inated appearance often showing the so-called basketweaving (shown in Fig. 11-13). The basket-weave appearance shows focal short fibrils of collagen at right angles to the longitudinal axis of the GBM. Subepithelial and subendothelial GBM scalloping can often be seen. Thin GBM segments may be absent as the disease progresses with the 
14

Fig. 14. X-linked Alport syndrome in a male patient with segmental thickened GBM $(\sim 800 \mathrm{~nm})$ with mottling and basketweave appearance (black arrow) and segmental thin GBM $(\sim 130 \mathrm{~nm})$ (white arrow) with moderate foot process effacement (transmission electron microscopy $\times 7,100$ ). GBM, glomerular basement membrane.

Fig. 15. X-linked Alport syndrome in a male patient with diffuse lamellation (black arrows) and basket-weave appearance $(\sim 700 \mathrm{~nm}$, thin areas $\sim 130 \mathrm{~nm})$ (white arrows) of the GBM with moderate foot process effacement (red arrows) (transmission electron microscopy $\times 2,200,00)$. GBM, glomerular basement membrane.

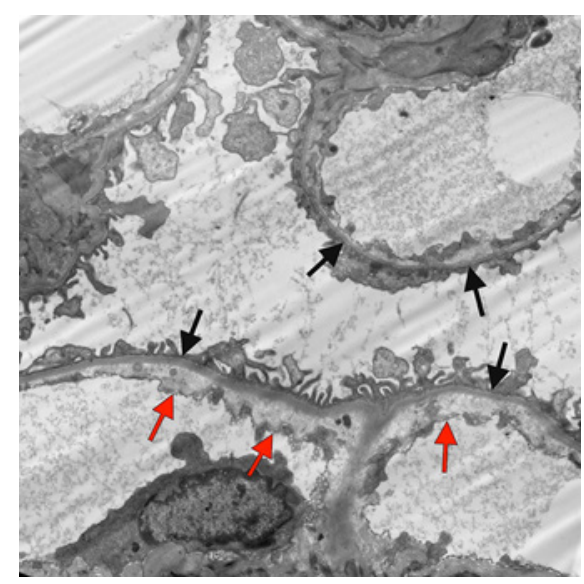

Fig. 16. Patient with thin GBM lesion $(\sim 100 \mathrm{~nm})$ (black arrows) and segmental expansion of lamina rara interna $(\sim 200 \mathrm{~nm}$ in these areas) (red arrows) due to chronic endothelial injury with largely intact foot processes with normal type IV collagen staining by IF (not shown) (transmission electron microscopy $\times 4,400$ ). GBM, glomerular basement membrane.

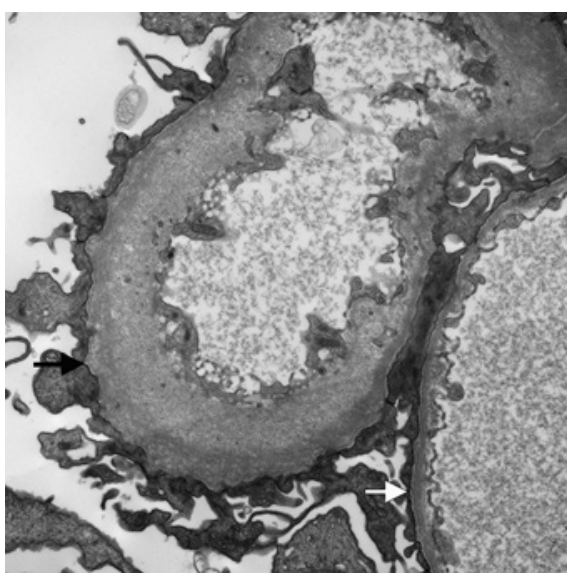

15
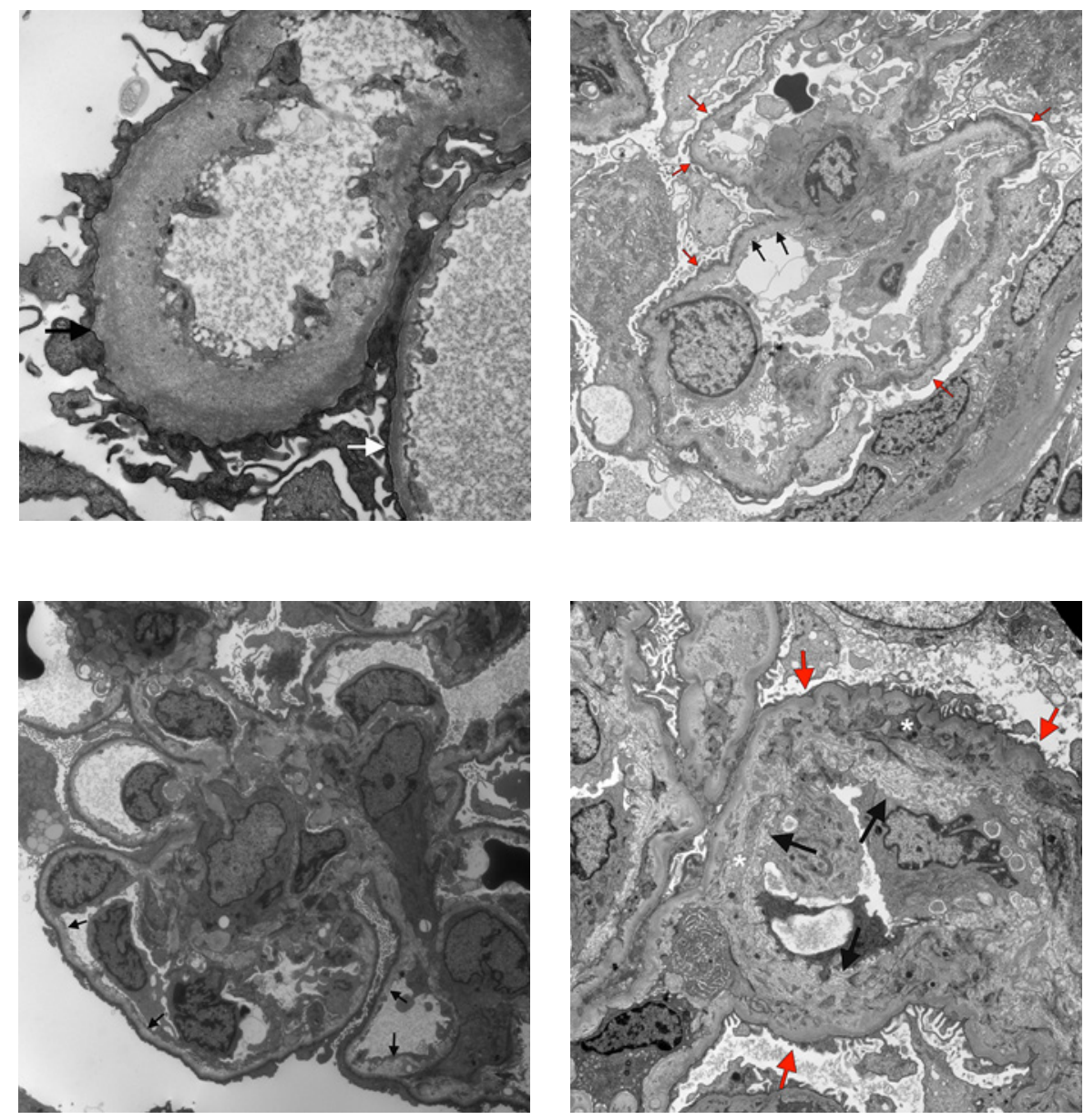

Fig. 17. Patient with thin GBM lesion $(\sim 150 \mathrm{~nm})$ and extensive mild expansion of lamina rara interna (arrows) due to chronic endothelial injury with normal type IV collagen staining by IF (not shown) with segmental foot process effacement (transmission electron microscopy $\times 2,200)$. GBM, glomerular basement membrane.
Fig. 18. Patient with severe expansion of lamina rara interna (black arrows) and cellular interposition (white asterisks) due to chronic endothelial injury, so-called chronic thrombotic microangiopathy, with segmental foot process effacement (red arrows) (transmission electron microscopy $\times 3,500)$. complete loss of the normal lamina densa and often with associated extensive effacement of visceral epithelial foot processes (shown in Fig. 14, 15). In some cases, partial visceral epithelial foot process effacement can occur with GBM changes. EM findings for Alport syndrome are not unique to a particular hereditary pattern; IF staining and/ or genetic studies as well as a detailed family history are needed to elicit the underlying genetic abnormality.

There are several diseases with ultrastructural GBM changes that can mimic Alport syndrome. All of these have a normal IF staining pattern for type IV collagen. In patients with Frasier syndrome the GBM is thin with split- ting and lamellation by EM. Frasier syndrome is caused by mutations involving WT1 (Intron 9). It presents with male pseudohermaphroditism, gonadal tumors, and progressive nephropathy. The patients show secondary focal segmental glomerulosclerosis with nephrotic syndrome usually in childhood [22]. In patients with Pierson syndrome, EM shows irregular, thick, and thin segments of the GBM with lamellation on the subepithelial and/or subendothelial sides. Pierson syndrome is caused by mutation in LAMB2 encoding laminin beta 2 and presents with nephrotic syndrome early in life, typically associated with microcoria. However, some patients may show re- 
19

Fig. 19. Patient with chronic membranous nephropathy and reabsorbed deposits (arrows) that give the GBM a thick and thin and mottled appearance ( 350-400 nm away from deposits) (transmission electron microscopy $\times 3,500)$. GBM, glomerular basement membrane.

Fig. 20. Adult male patient status post kidney en bloc pediatric donor transplant from a 2-month-old infant with thin GBM (normal for infant age $\sim 100 \mathrm{~nm}$, areas with interposition $\sim 800 \mathrm{~nm}$ ) with segmental early lamellation, mottling and scalloping (arrows) (transmission electron microscopy $\times 8,900$ ). GBM, glomerular basement membrane.

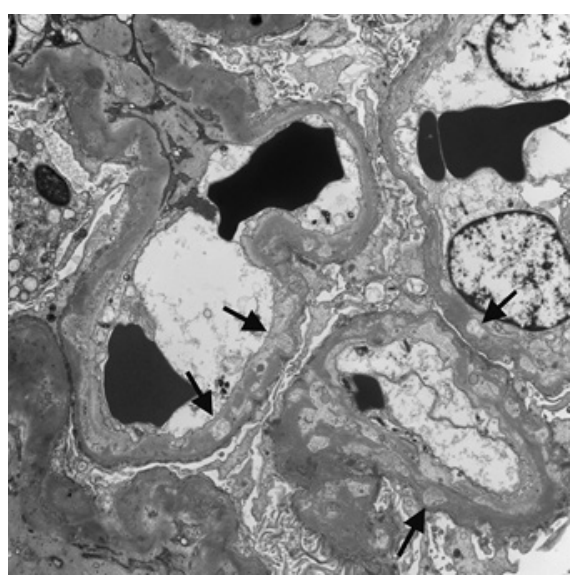

20

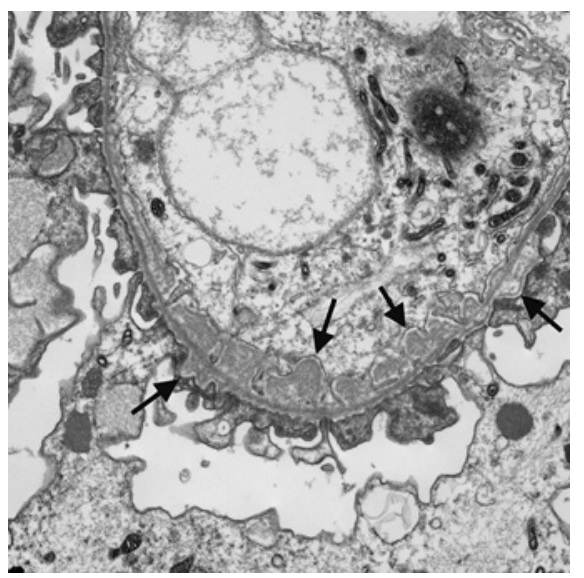

nal-limited disease. By light microscopy, increased mesangial matrix and global glomerulosclerosis can be seen $[23,24]$. Nail-patella syndrome is caused by LMX1B mutation, with associated nail, elbow, iliac horn, and patellar abnormalities. Many patients also develop proteinuria in the young adulthood. EM shows irregular thickening of glomerular capillary walls with a moth-eaten appearance. Special staining techniques unveil within the "moth-eaten" defects type III collagen bundles that may give rise to a pattern of basket-weaving [25]. The defects and collagen type III deposits are often noted within the lamina densa. Diseases resulting from chronic endothelial injury, that is, chronic thrombotic microangiopathy or transplant glomerulopathy, show primarily an expansion of the lamina rara interna, but no abnormalities along the lamina densa perse (shown in Fig. 16-18). Immune complex-mediated glomerulonephritides with signs of resolution present with foci of electron lucency within the capillary walls and structural irregularities that can mimic HN (shown in Fig. 19). However, usually a correct diagnosis of "glomerulonephritis" can be rendered based on LM and IF findings (including a normal staining pattern for collagen IV subtypes), including IgA nephropathy associated with thick, thin, and/or irregular GBMs. Pediatric (particularly small infant) donor kidneys implanted into the adult recipients can lead to glomerular injury, so-called size-mismatch glomerulopathy or pediatric donor glomerulopathy, caused by stress adaptation of the GBM (shown in Fig. 20). The ultrastructural appearance of a size mismatch glomerulopathy mimics Alport disease; however, the structural irregularities of the capillary walls, including thickening, lamellations, and scalloping, affect the subepithelial side of the GBM; the lamina densa typically remains identifiable and intact (see HN) [26].
Thin GBM lesion is diagnosed when there is extensive thinning of the GBM without other structural irregularities of the lamina densa. Thin basement membrane lesion can in some cases represent a heterozygous state of Alport gene mutations with incomplete penetrance or it might be found in very early phases of $\mathrm{HN}$.

\section{Summary}

Diagnosis of HN/thin GBM morphologically is most optimally done by EM which shows characteristic findings of thin and/or abnormal thickened segments with basket-weaving. In some cases characteristic IF staining patterns for alpha 5 type IV collagen can confirm the diagnosis of COL4 nephropathy. In other patients, next generation sequencing may be necessary to diagnose the specific etiology of the abnormal GBM.

\section{Conflict of Interest Statement}

The authors have no conflicts of interest to declare.

\section{Funding Sources}

The authors have no funding sources to declare.

\section{Author Contributions}

Outline and concepts: M.A.L. and A.B.F. Draft writing: M.A.L. Final editing and organization: M.A.L. and A.B.F. 


\section{References}

1 Vivante A, Calderon-Margalit R, Skorecki K. Hematuria and risk for end-stage kidney disease. Curr Opin Nephrol Hypertens. 2013 May;22(3):325-30.

2 Gubler MC. Inherited diseases of the glomerular basement membrane. Nat Clin Pract Nephrol. 2008 Jan;4(1):24-37.

3 Tryggvason K, Patrakka J. Thin basement membrane nephropathy. J Am Soc Nephrol. 2006 Mar;17(3):813-22.

4 Heidet L, Gubler MC. The renal lesions of Alport syndrome. J Am Soc Nephrol. 2009 Jun; 20(6):1210-5.

5 Morinière $\mathrm{V}$, Dahan K, Hilbert $\mathrm{P}$, Lison $\mathrm{M}$, Lebbah S, Topa A, et al. Improving mutation screening in familial hematuric nephropathies through next generation sequencing. J Am Soc Nephrol. 2014 Dec;25(12):2740-51.

6 Fallerini C, Dosa L, Tita R, Del Prete D, Feriozzi S, Gai G, et al. Unbiased next generation sequencing analysis confirms the existence of autosomal dominant Alport syndrome in a relevant fraction of cases. Clin Genet. 2014 Sep;86(3):252-7.

7 Gast C, Pengelly RJ, Lyon M, Bunyan DJ, Seaby EG, Graham N, et al. Collagen (COL4A) mutations are the most frequent mutations underlying adult focal segmental glomerulosclerosis. Nephrol Dial Transplant. 2016 Jun; 31(6):961-70

8 Savige J, Colville D, Rheault M, Gear S, Lennon $\mathrm{R}$, Lagas $\mathrm{S}$, et al. Alport syndrome in women and girls. Clin J Am Soc Nephrol. 2016 Sep;11(9):1713-20.

9 Kashtan CE. Alport syndrome. In: Adam MP, Ardinger HH, Pagon RA, Wallace SE, Bean LJH, Mirzaa GM, et al., editors. GeneReviews [Internet]. Seattle, WA: University of Washington. 2001 Aug 28. [Updated 2019 Feb 21]. Available from: https://www.ncbi.nlm.nih.gov/ books/NBK1207/ Accessed 2019 March 27.
10 Temme J, Kramer A, Jager KJ, Lange K, Peters F, Müller GA, et al. Outcomes of male patients with Alport syndrome undergoing renal replacement therapy. Clin J Am Soc Nephrol. 2012 Dec;7(12):1969-76.

11 Storey H, Savige J, Sivakumar V, Abbs S, Flinter FA. COL4A3/COL4A4 mutations and features in individuals with autosomal recessive Alport syndrome. J Am Soc Nephrol. 2013 Dec;24(12):1945-54.

12 Imafuku A, Nozu K, Sawa N, Hasegawa E, Hiramatsu R, Kawada M, et al. Autosomal dominant form of type IV collagen nephropathy exists among patients with hereditary nephritis difficult to diagnose clinicopathologically. Nephrology. 2018 Oct;23(10):940-7.

13 Spetie DN, Nadasdy T, Nadasdy G, Agarwal G, Mauer M, Agarwal AK, et al. Proposed pathogenesis of idiopathic loin pain-hematuria syndrome. Am J Kidney Dis. 2006 Mar; 47(3):419-27.

14 Savige J. Alport syndrome: About time - treating children with Alport syndrome. Nat Rev Nephrol. 2012 May;8(7):375-6.

15 Kashtan CE, Ding J, Gregory M, Gross O, Heidet L, Knebelmann B, et al. Clinical practice recommendations for the treatment of Alport syndrome: a statement of the Alport Syndrome Research Collaborative. Pediatr Nephrol. 2013 Jan;28(1):5-11.

16 Savige J, Gregory M, Gross O, Kashtan C, Ding J, Flinter F. Expert guidelines for the management of Alport syndrome and thin basement membrane nephropathy. J Am Soc Nephrol. 2013 Feb;24(3):364-75.

17 Foster K, Markowitz GS, D'Agati VD. Pathology of thin basement membrane nephropathy. Semin Nephrol. 2005 May;25(3):149-58.

18 Raju P, Cimbaluk D, Korbet SM. The variable course of women with X-linked Alport syndrome. Clin Kidney J. 2013 Dec;6(6):630-4.
19 Kagawa M, Kishiro Y, Naito I, Nemoto T, Nakanishi H, Ninomiya Y, et al. Epitope-defined monoclonal antibodies against type-IV collagen for diagnosis of Alport's syndrome. Nephrol Dial Transplant. 1997 Jun;12(6): 1238-41.

20 Nasr SH, Markowitz GS, Valeri AM, Yu Z, Chen L, D'Agati VD. Thin basement membrane nephropathy cannot be diagnosed reliably in deparaffinized, formalin-fixed tissue. Nephrol Dial Transplant. 2007 Apr;22(4): 1228-32.

21 Haas M. Alport syndrome and thin glomerular basement membrane nephropathy: A practical approach to diagnosis. Arch Pathol Lab Med. 2009 Feb;133(2):224-32.

22 Ito S, Hataya $\mathrm{H}$, Ikeda $\mathrm{M}$, Takata $\mathrm{A}$, Kikuchi $\mathrm{H}$, Hata J, et al. Alport syndrome-like basement membrane changes in Frasier syndrome: An electron microscopy study. Am J Kidney Dis. 2003 May;41(5):1110-5.

23 Kagan M, Cohen AH, Matejas V, Vlangos C, Zenker M. A milder variant of Pierson syndrome. Pediatr Nephrol. 2008;23(2):323-7.

24 Choi HJ, Lee BH, Kang JH, Jeong HJ, Moon $\mathrm{KC}, \mathrm{Ha}$ IS, et al. Variable phenotype of Pierson syndrome. Pediatr Nephrol. 2008 Jun; 23(6):995-1000.

25 Harita Y, Kitanaka S, Isojima T, Ashida A, Hattori M. Spectrum of LMX1B mutations: from nail-patella syndrome to isolated nephropathy. Pediatr Nephrol. 2017 Oct;32(10): 1845-50.

26 Nadasdy T, Abdi R, Pitha J, Slakey D, Racusen L. Diffuse glomerular basement membrane lamellation in renal allografts from pediatric donors to adult recipients. Am J Surg Pathol. 1999 Apr;23(4):437-42. 